\title{
Development of Metrics for Resilience Quantification in Energy Systems
}

\author{
Fellipe Sartori da Silva ${ }^{1}$, José Alexandre Matelli ${ }^{2}$ \\ ${ }^{1,2}$ São Paulo State University, Department of Energy, Guaratinguetá, São Paulo, 12516-410, Brazil \\ fellipe.sartori@unesp.br \\ jose.a.matelli@unesp.br
}

\begin{abstract}
The design of energy systems usually requires technical, economical and environmental analysis. However, the growth of systems failure due to unpredictable lowprobability external events makes the consideration of resilience in this design also important. Although there is no standard metric for resilience quantification yet, it is known that it should consider system configuration, operation time and total or partial energy generation during and after the event, as well as the components repair probability and time. A proposal for resilience quantification in four cogeneration plants was previously developed based on components stochastic failures and verification of their consequences in the plant energy generation. The present work aims to continue the development of this metric by including in its calculation the repair probability of the components, their repairing time and the plant downtime during the repair, essential parameters for resilience quantification. Two new metrics are proposed and simulations with $0,50 \%$ and $75 \%$ of repair probability of the components are made in software CLIPS. One of the metrics is able to evaluate the influence of repairment in system resilience, while the other one predicts plant downtime during operation. The metrics point to $\mathrm{S} \# 2$ as the most resilient system and S\#3 as the most affect by repairing.
\end{abstract}

\section{INTRODUCTION}

The development of actual society is fully dependent on energy, which has its demand continuously increased over time. Energy systems have been studied technically and structurally, in order to enhance power supply and increase both efficiency and reliability.

Regarding technical aspects, the design of energy systems usually considers energy balance, as well as economical and

Fellipe Sartori da Silva et al. This is an open-access article distributed under the terms of the Creative Commons Attribution 3.0 United States License, which permits unrestricted use, distribution, and reproduction in any medium, provided the original author and source are credited. environmental analysis. In this context, power plants working with cogeneration technology stand out due to their high conversion efficiency and capacity to generate power and useful thermal energy (cooling or heating) simultaneously from a single fuel source (Silva, Matelli and Bazzo, 2014). Additionally, these systems have flexibility to operate in several places such as industries, residential buildings and hospitals (Isa, Tan and Yatim, 2018). Although many equipment can be used as prime movers, internal combustion engines and gas turbines are currently the most attractive, since they have commercial advantages when compared to other components (Matelli \& Goebel, 2018).

Structural analyses of energy systems depend mainly on reliability concepts, which consider expected events to predict failure of components and, consequently, of the whole plant. However, the growth of failures due to unpredictable low-probability high-impact external events in the last years increased the structural vulnerability of these systems (Shen, Gut and Zhao, 2019). To evaluate the consequences of such events, a recent approach focused on resilience has been introduced.

Resilience can be defined as the ability of the system to operate totally or partially at some functional level under scenarios before, during and after low-probability highimpact events. It also includes its capacity to withstand and recover quickly from unexpected disruptions (Sandia National Laboratories, 2014).

According to Hickford, Blainey, Hortelano and Pant (2018) and Francis and Bekera (2014), a resilient system must be able to absorb, adapt and recover from unpredictable situations. The absorptivity is related with the impact that the system can withstand before its partial or total disruption, as long as the recovery capacity is related to how rapidly the system can return to its partial or total functional operation after the event. The adaptation is the ability of the system to change some features in its structure in response to an adverse situation caused by an event, in order to continue its operation at some functional level. 
As a relative new concept, there is no standard measurement framework for resilience in energy systems (Lin \& Bie, 2016). Some authors proposed metrics focused either on estimate resilience value, compare this parameter for different configurations and analyze the consequence of system disruption. As examples, Mehrpouyan, Haley, Dong, Tumer and Hoyle (2015) developed a comparative model with focus on features of components and the system; Yodo and Wang (2016) proposed a metric based on analytical methods taking into account characteristics of the disruption and system reliability; Martisauskas, Augutis and Krikstolaitis (2018) analyzed conditions since before the event, like threats and possible disruptions, until consequences in energy cost and supply to propose an security coefficient; Hossain, Jaradat, Hosseini, Marufuzzaman and Buchanan (2019) quantified absorptive, adaptive and restorative capacities in a Bayesian network and compared with a recovered lost production capacity to estimate system resilience.

A previously framework was developed by Matelli and Goebel (2018), on which five resilience metrics were proposed through simulation of stochastic failures within a Monte Carlo-based assessment approach for four different configurations of cogeneration systems. Their results pointed to a convergence of all metrics to the same sequence of systems' higher values of resilience. However, some characteristics related to repair probability of the components are also important to this quantification, as stated in Sandia Report (Sandia National Laboratories, 2014). The aim of the present work is to continue the development of this method of resilience quantification by considering the repair probability of the components, their repairing time and the plant downtime during the repair. We hypothesize that those parameters are strongly related to the system recovery capacity. Therefore, the contribution of this work is to propose new resilient metrics that should reflect this condition.

\section{Methodology}

\subsection{Systems description}

As described by Matelli and Goebel (2018), four cogeneration systems (S\#) were previously generated by a knowledge-based system (KBS) developed by Silva et al. (2014). The considerations required for this development are expressed in Table 1; Figures 1-4 illustrate the proposed plants.

Table 1. Considerations required for systems development

\begin{tabular}{ll}
\hline Parameter & Value \\
\hline Local average temperature $\left[{ }^{\circ} \mathrm{C}\right]$ & 18 \\
Local altitude $[\mathrm{m}]$ & 670 \\
Maximum power demand $[\mathrm{kW}]$ & 1500 \\
Minimum power demand $[\mathrm{kW}]$ & 900 \\
Electrical energy consumption [MWh] & 28 \\
Chilled water demand [kW] & 1407 \\
Daily operation [h/day] & 24 \\
Weekly operation [days/week] & 7 \\
Electric connection scheme & Tied to the grid \\
Chilled water storage & No \\
\hline
\end{tabular}

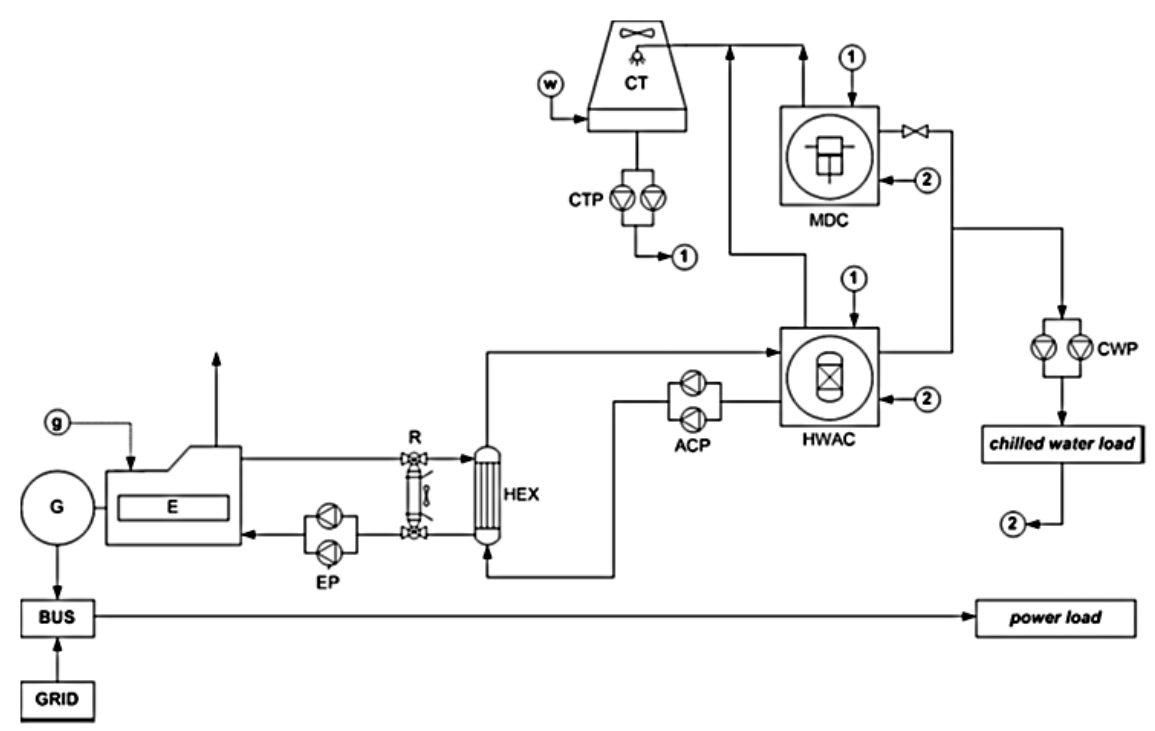

Figure 1. S\#1: system based on one internal combustion engine generation 


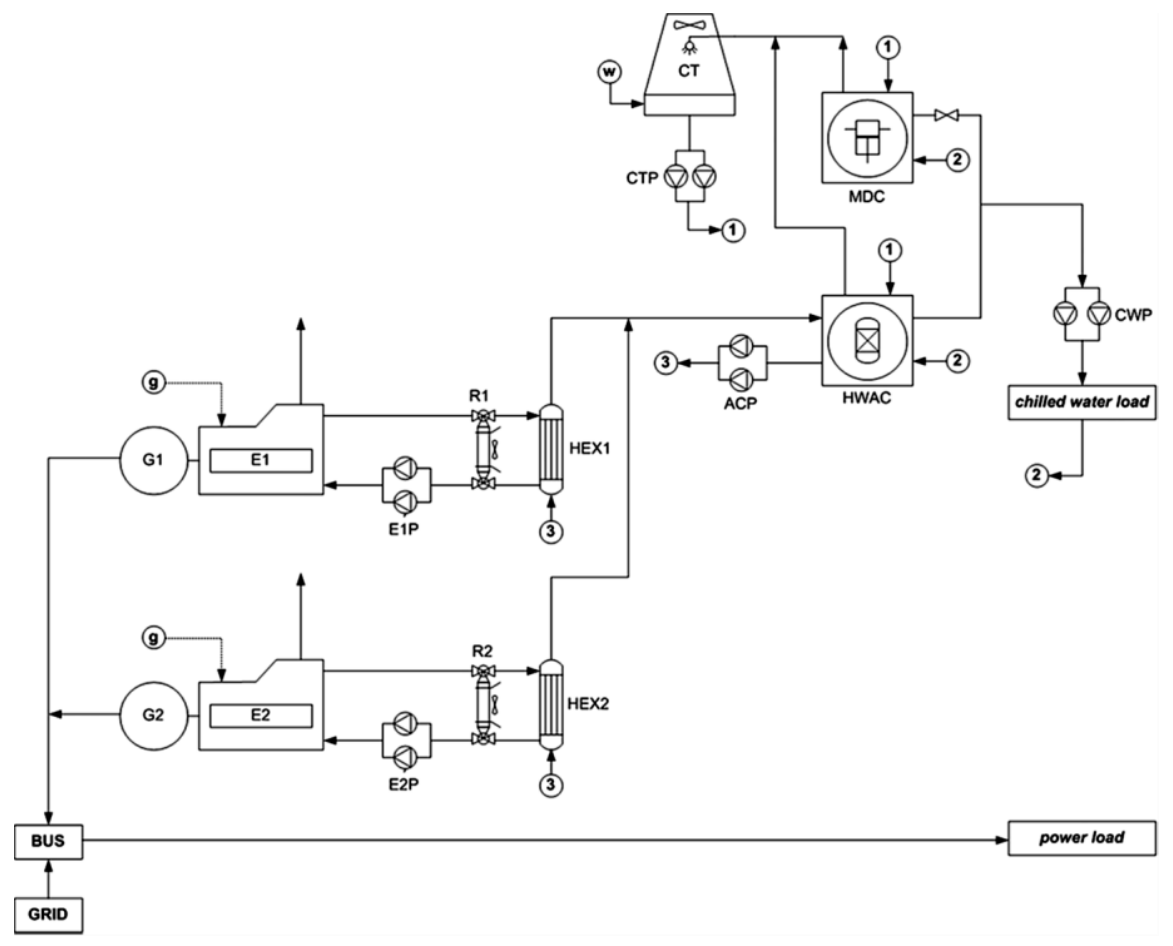

Figure 2. S\#2: system based on two internal combustion engine generation

As it can be seen, the capital difference between $\mathrm{S \# 1}$ and $\mathrm{S \# 2}$ is the number of internal combustion engines (E) coupled to the respective generator $(\mathrm{G})$, which is connected to a bus bar along with the external grid, meeting the previously requested power load. A heat exchanger (HEX) is used to produce hot water from the heat associated to the engine jacket water. The produced hot water feeds a single effect absorption chiller (HWAC), in order to produce enough chilled water as demanded. A radiator $(\mathrm{R})$ is connected to the engine to keep it operating when the HWAC is off. A mechanical-driven chiller (MDC) can suit as supplement or backup. Lastly, a cooling tower (CT) rejects the heat released from both chillers.

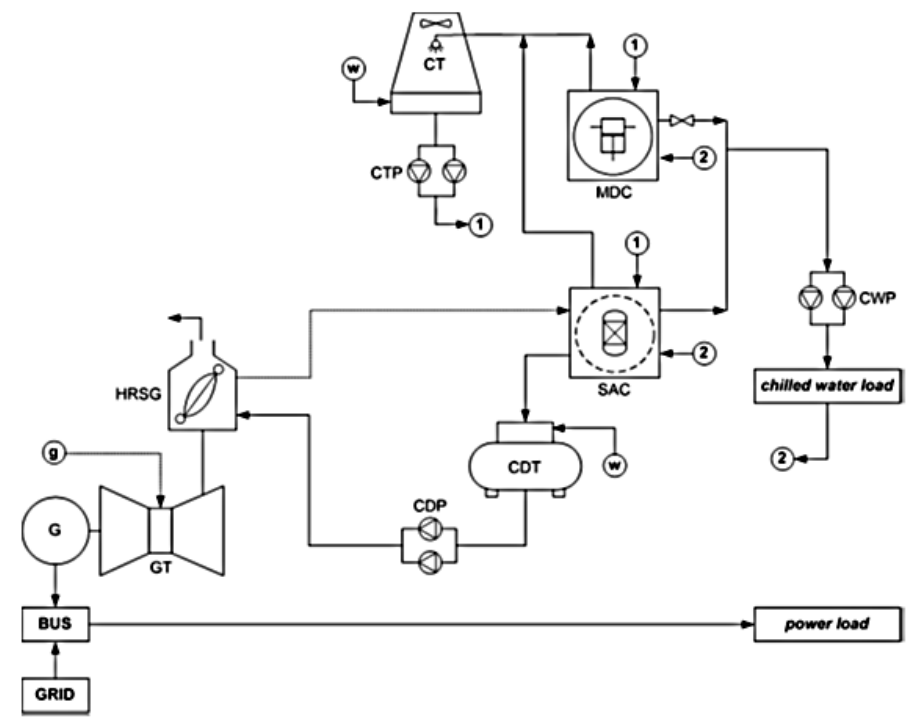

Figure 3. S\#3: system based on one gas turbine generation 


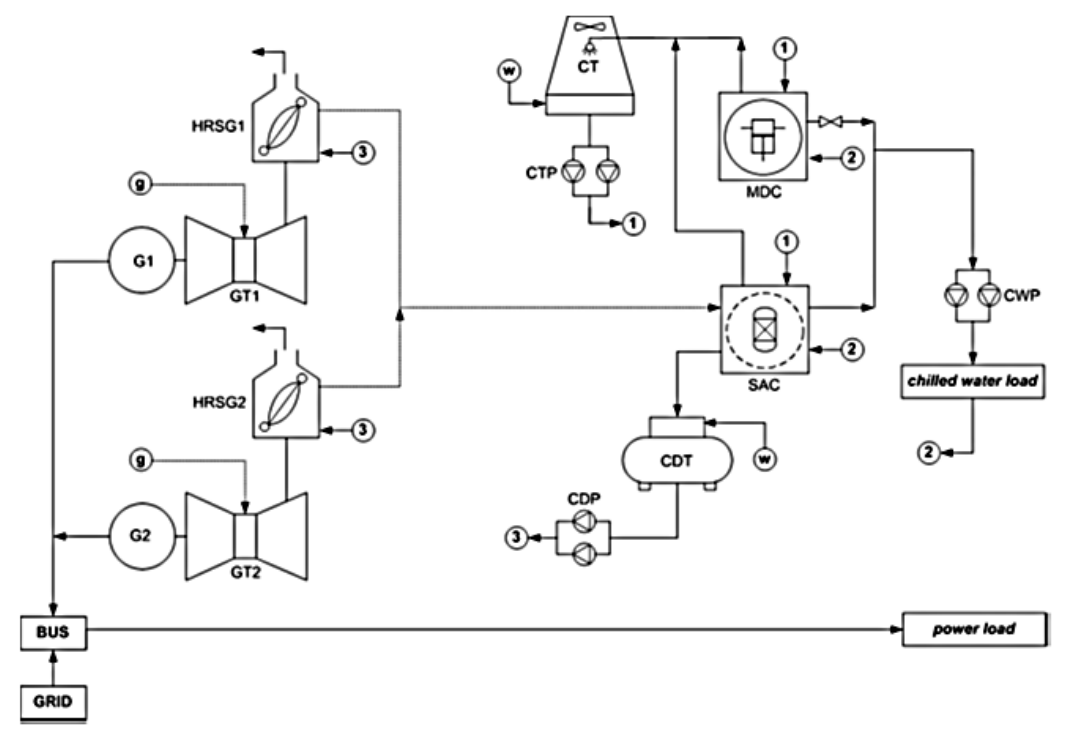

Figure 4. S\#4: system based on two gas turbines generation

As in the internal combustion engine-based systems, S\#3 and $\mathrm{S \# 4}$ also differ by the number of prime movers, in these cases a gas turbine (GT). GT is coupled to a generator (G), which feeds the bus bar along with the external grid, in order to meet the specified power load. GT exhaust gases reject heat in a heat recovery steam generator (HRSG), where steam is produced to feed a double effect absorption chiller (SAC), in order to meet the requested chilled water demand. A mechanical-driven chiller (MDC) can suit as backup. Finally, a cooling tower (CT) rejects the heat released from both chillers.

Information about systems thermal performances can be found in Silva et al. (2014).

\subsection{Simulation framework}

The four cogeneration plants are modeled in software ' $C$ ' Language Integrated Production System (CLIPS), specifically designed to develop knowledge-based systems. Simulations of stochastic failure of components are performed with Monte Carlo approach, i.e., with several repetitions at the same conditions in order to verify standard operation responses for each configuration.

The simulations proceed as follows: a non-failed component $i$ is randomly chosen as a candidate to fail. A failure probability $\left(p_{b}\right)$ is randomly assigned and compared to a known probability of component normal operation $\left(p_{i}\right)$. If $p_{b}$ $>p_{i}$, the component $i$ fails, otherwise a new choice is made. In case of failure, all the components connected to the component $i$ and to the propagated failed components are checked, in order to verify the failure propagation throughout the system. Failure propagation rule is illustrated in Figure 5.

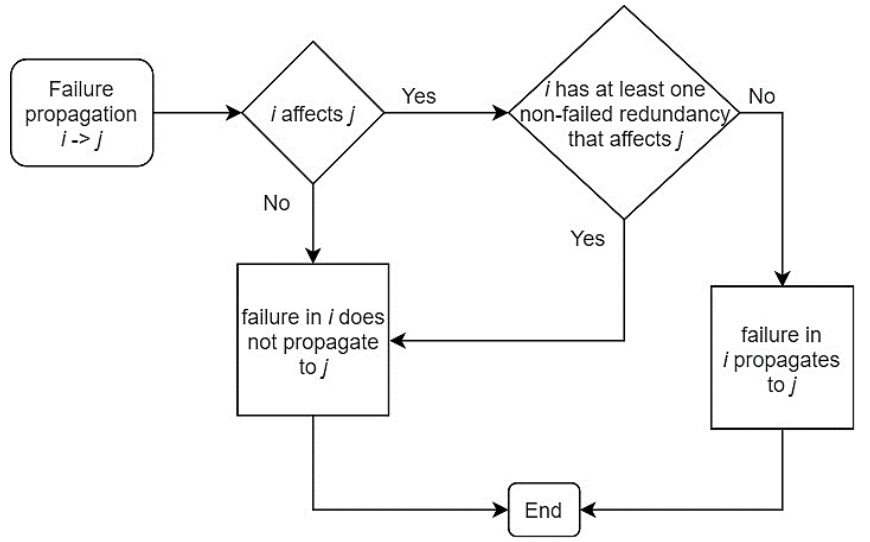

Figure 5. Failure propagation rule

Once the failure propagation is verified, a repair probability $\left(p_{c r}\right)$ is randomly assigned and compared to a known nonviability repair probability $\left(p_{c n r}\right)$. If $p_{c r}>p_{c n r}$, component $i$ starts the repair process, otherwise it keeps failed until the end of the simulation. In repairing processes, the time spent in repair $(t s)$ increases in a unity every hour of plant operation until it reaches the repair time (rt). Then, the component $i$ and all the components that failed by propagation return to their non-failed condition.

Considering all scenarios of failure throughout the simulation, the functional state of the system can be classified in three ways:

- Normal: the system has no failed components and, therefore, meet power and chilled water demand completely; 
- Resilient: the system has failed components, but it is still able to meet partially at least one of the demands;

- Failed: the system can no longer generate power and chilled water.

Based on these functional states, four time counters are proposed:

- $\quad$ Normal time $(n t)$ : time in which the system operates in its normal state. It indicates operation with no failures when $n t=T$;

- Resilient time $(r)$ : time counting for system resilient operation. It points to instantaneously system failure or normal operation when $r=0$ and to resilient operation when $0<r<t$;
- Downtime $(d)$ : time in which the system is failed and has repairing components. When a component is completely repaired and it consequently leads to a return to normal or resilient operation, $d$ stops being counted. It indicates system with uninterrupted operation until failure or lifetime reach without repairment when $d=0$;

- Simulation time $(t)$ : System total operation time, including resilient, normal and downtime. System failure occurs when $t<T$ and lifetime reach occurs when $t=T$.

In order to illustrate the methodology proposed in this work, with all the time counters and processes described above, Fig. 6 presents a flow chart of the simulation steps.

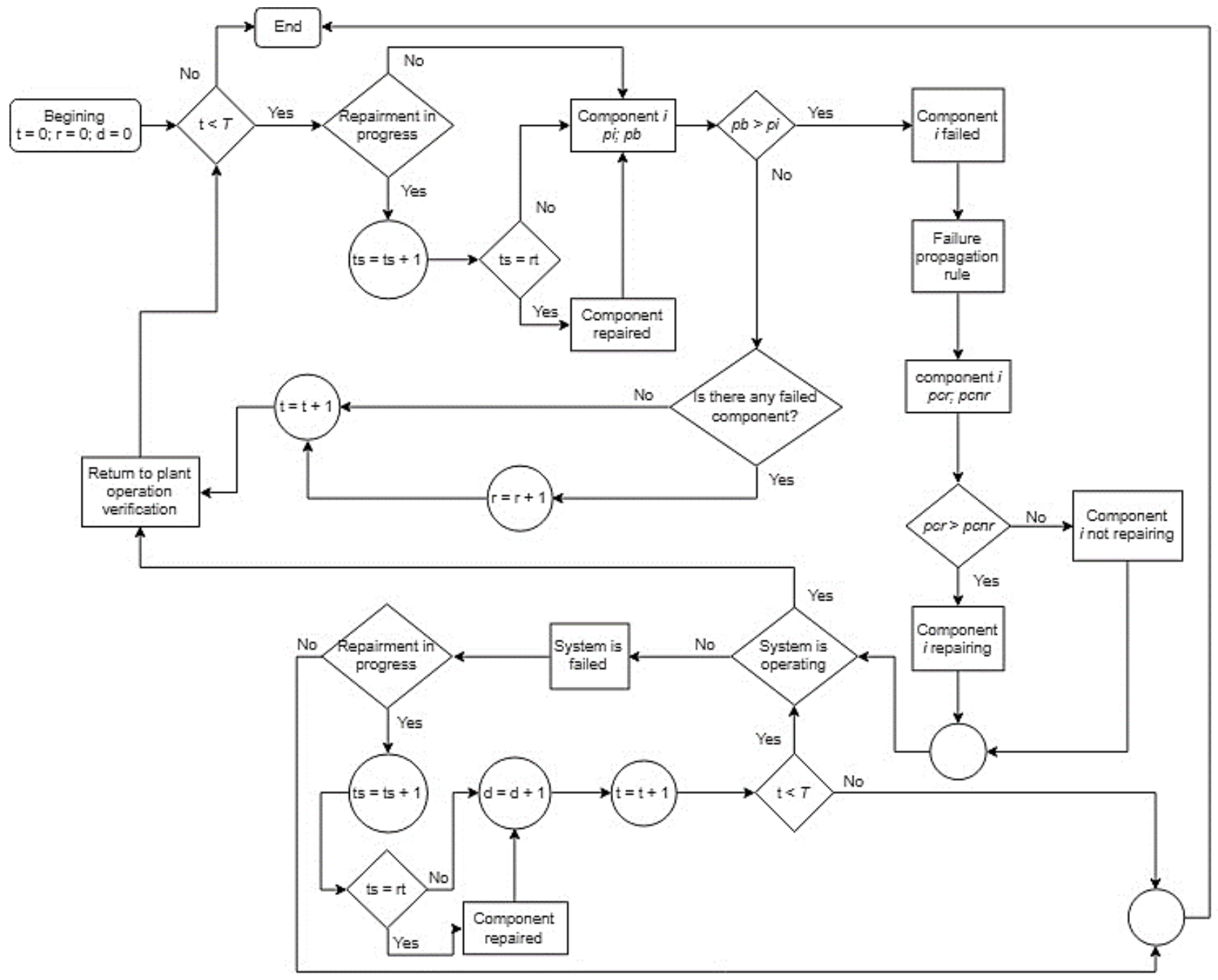

Fig 6. Flow chart of proposed methodology 
The simulation requires two parameters to start: system expected operation time, $T$, and number of simulations, $N$.

The proposed modeling considers the following assumptions:

- $T=8760 \mathrm{~h}$, simulating a whole year of uninterrupted operation, and $N$ is fixed in 3000 simulations, enough to stabilize the coefficient of variation (Matelli \& Goebel, 2018);

- All the components have constants and timeindependent $p_{i}$ and $p_{c n}$;

- Each component repairing time follows the values assumed in Table 2;

- Failure propagation considers all the components connected to original failed one, regardless of the nature of the connection, with constant and time-independent propagation probability equal to 1 ;

- Component functional state is either failed or normal, with no partial operation admitted.

The value of $p_{i}$ is assumed to be 0.9995 as in Matelli and Goebel (2018), focusing on results comparison, as long as it was assigned three different values to $p_{c n r}: 1,0.50$ and 0.25 . The first value is assigned to keep the simulation with no repair probability, in order to compare the results with the previously work; the second one intends to keep the component repairing at chance (i.e., the repairing success is no better than a flip coin); the third value leads to greater repair probability, which provides clearer information about the influence of the repairing success in the system resilience. Repair times shown in Table 2 were assumed considering components function in order to standardize proportional values.

Table 2. Components repairing time

\begin{tabular}{|c|c|c|}
\hline Function & $\begin{array}{l}\text { Repairing } \\
\text { time (h) }\end{array}$ & Component \\
\hline Shaft work generation & 100 & $\begin{array}{l}\text { Turbine } \\
\text { Engine }\end{array}$ \\
\hline Power generation & 80 & Generator \\
\hline Heat exchange & 60 & $\begin{array}{l}\text { Hot water absorption chiller } \\
\text { Mechanical chiller } \\
\text { Heat exchanger } \\
\text { Steam absorption chiller } \\
\text { Heat recovery steam generator } \\
\text { Cooling tower } \\
\text { Radiator } \\
\end{array}$ \\
\hline Feeding & 40 & $\begin{array}{l}\text { Chilled water pump } \\
\text { Hot water chiller pump } \\
\text { Jacket water pump } \\
\text { Cooling tower pump } \\
\text { Condensate pump }\end{array}$ \\
\hline Structural & 40 & $\begin{array}{l}\text { Grid } \\
\text { Bus } \\
\text { Power load } \\
\text { Chilled water load } \\
\text { Gas line } \\
\text { Water line } \\
\end{array}$ \\
\hline Storage & 30 & Condensate tank \\
\hline
\end{tabular}

\subsection{Metrics for resilience quantification}

Considering the simulation framework previously described, two new metrics (vi and vii) are proposed in this work to complement the five metrics (i-v) developed by Matelli and Goebel (2018).

i. Fraction of simulations with resilient operation $\left(p_{r}\right)$ : ratio between the number of simulations $k$ in which $t_{k}=T$ and $0<r<t_{k}\left(N_{r}\right)$ and the total number of simulations $N$. It can be interpreted as the probability of the system to operate at resilient functional state. The higher $p_{r}$, the higher the system resilience.

$$
p_{r}=\lim _{N \rightarrow \infty}\left(\frac{N_{r}}{N}\right)
$$

ii. Resilient operating time $(\bar{r})$ : weighted average of the resilient operating time $r$ for all $N_{r}$ simulations, in which $t_{k}=T$ and $0<r<t_{k}$. It indicates which system can operate in a resilient condition for longer periods.

$$
\bar{r}=\frac{p_{r}}{N_{r}} \sum_{k=1}^{N_{r}} r_{k}
$$

iii. Time until failure $(\bar{f})$ : average time for all simulations $k$ in which $t_{k}<T$ and $r_{k}<t_{k}$, i.e., all simulations that result in a total system failure before admitted lifetime $\left(N_{f}\right)$. Higher value of this parameter indicate system with enhanced resilience.

$$
\bar{f}=\frac{1}{N_{f}} \sum_{k=1}^{N_{f}} t_{k}
$$

iv. Fraction of simulations with completely failed operation $\left(p_{f}\right)$ : relation between the number of simulations $k$ in which $t_{k}<T$ and $r_{k}<t_{k}\left(N_{f}\right)$ and the total number of simulations $N$. It can be interpreted as the probability of the system to fail. Systems with lower resilience exhibit high $p_{f}$.

$$
p_{f}=\lim _{N \rightarrow \infty}\left(\frac{N_{f}}{N}\right)
$$

v. Normalized resilience index $(\rho)$ : an average operating time $(\bar{t})$ is firstly calculated considering the system failure probability $\left(p_{f}\right)$ and its complementary value, as expressed in Eq. 5. Therefore, the parameter $\bar{t}$ is a weighted average between the time in simulations in which the system fails completely and the time in simulations that result in operation until its admitted lifetime. Then, $\bar{t}$ is normalized regarding $T$ (Eq. 6).

$$
\bar{t}=p_{f} \bar{f}+\left(1-p_{f}\right) T
$$




$$
\rho=\frac{\bar{t}}{T}
$$

The closer $\rho$ is to the unity, the higher the system resilience.

vi. Fraction of simulation with system recovery $\left(p_{d}\right)$ : ratio between the number of simulations $k$ in which $t_{k}=T$ and $d_{k}>0\left(N_{d}\right)$ and the total number of simulations $N$.

$$
p_{d}=\lim _{N \rightarrow \infty}\left(\frac{N_{d}}{N}\right)
$$

A higher number of $k$ simulations that result in system failure with recuperation $\left(N_{d}\right)$ indicates a higher number of failures during the operation, but with high rate of successful repairs. In the limit case where all repairing actions are unsuccessful, the value of $p_{d}$ gets closer to $p_{f}$ when no repairing actions are taken, i.e., $p_{d} \approx p_{f}$. Therefore, the higher the difference $p_{f}-p_{d}$, the higher the impact of repairing actions on the system resilience.

vii. System downtime $(\bar{D})$ : weighted average of the plant downtime $d$ for all $k$ simulations with $t_{k}=T$ and $d_{k}>0$ that result in system failure with recovery $\left(N_{d}\right)$. It indicates how long the system remains failed before return to normal operation. Therefore, the lower this parameter, the higher system resilience.

$$
\bar{D}=\frac{p_{d}}{N_{d}} \sum_{k=1}^{N_{d}} d_{k}
$$

\section{RESULTS AND DISCUSSION}

Although thermal performance and system costs are not discussed herein, Silva et al. (2014) found that from that perspective, the preferred system choice would be S\#1, followed by $\mathrm{S} \# 2, \mathrm{~S} \# 3$ and $\mathrm{S} \# 4$, respectively. Regarding initially proposed resilience metrics, the numerical results that Matelli and Goebel (2018) (represented in Table 3 as $\mathrm{M} \& \mathrm{G})$ found in their work with simulations considering no repairing actions are compared with the results of this work considering $p_{c n r}=1$, i.e., also with no repairment. The comparisons are represented in Table 3.

Table 3. Comparisons of results from Matelli and Goebel (2018) with this work with no repairing.

\begin{tabular}{lllll}
\hline Parameter/System & S\#1 & S\#2 & S\#3 & S\#4 \\
\hline$p_{r}(\mathrm{M} \& \mathrm{G})$ & 0.516 & 0.654 & 0.456 & 0.568 \\
$p_{r}$ & 0.490 & 0.638 & 0.453 & 0.581 \\
\hline $\bar{r}(\mathrm{M} \& \mathrm{G})$ & 3188 & 4245 & 2890 & 3619
\end{tabular}

\begin{tabular}{lllll}
$\bar{r}$ & 3013 & 4097 & 2722 & 3635 \\
\hline $\bar{f}(\mathrm{M} \& \mathrm{G})$ & 5057 & 5198 & 4849 & 5116 \\
$\bar{f}$ & 5038 & 5317 & 4908 & 5049 \\
\hline$p_{f}(\mathrm{M} \& \mathrm{G})$ & 0.461 & 0.328 & 0.526 & 0.414 \\
$p_{f}$ & 0.482 & 0.338 & 0.525 & 0.396 \\
\hline$\rho(\mathrm{M} \& \mathrm{G})$ & 0.805 & 0.867 & 0.765 & 0.828 \\
$\rho$ & 0.795 & 0.867 & 0.769 & 0.832 \\
\hline
\end{tabular}

As it can be noted in Table 3, all the metrics in both works point to system $\mathrm{S} \# 2$ as the most resilient, followed by $\mathrm{S \# 4}$, $\mathrm{S \# 1}$ and S\#3, respectively. With no repairing actions, it is clear that redundancy is the most impactful factor in resilience value.

The results of the seven metrics proposed in this work with $p_{c n r}=0.25,0.5$ and 1 are shown in Figures 7-13. Compared to results represented in Table 3, values presented in these figures indicate that repairing actions increase system resilience, as expected.

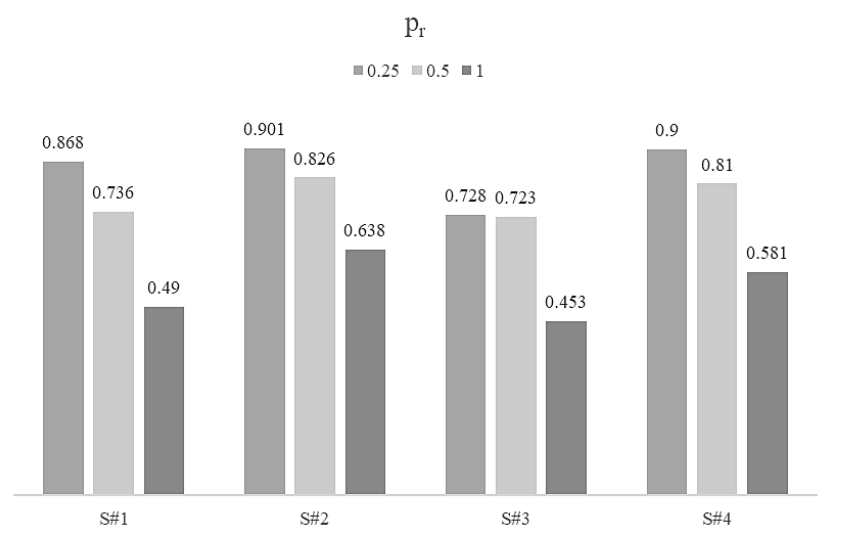

Fig 7. Variation of $p_{r}$ with different $p_{c n r}$ values

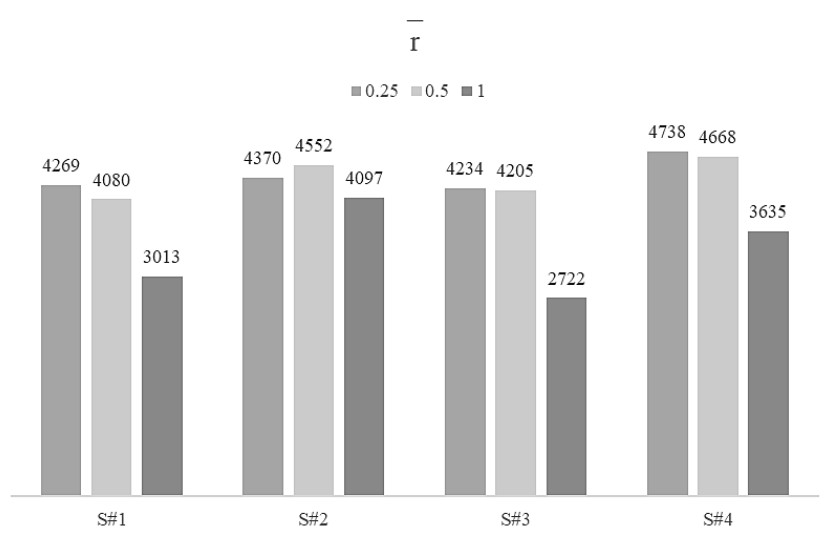

Fig 8. Variation of $\bar{r}$ with different $p_{c n r}$ values 


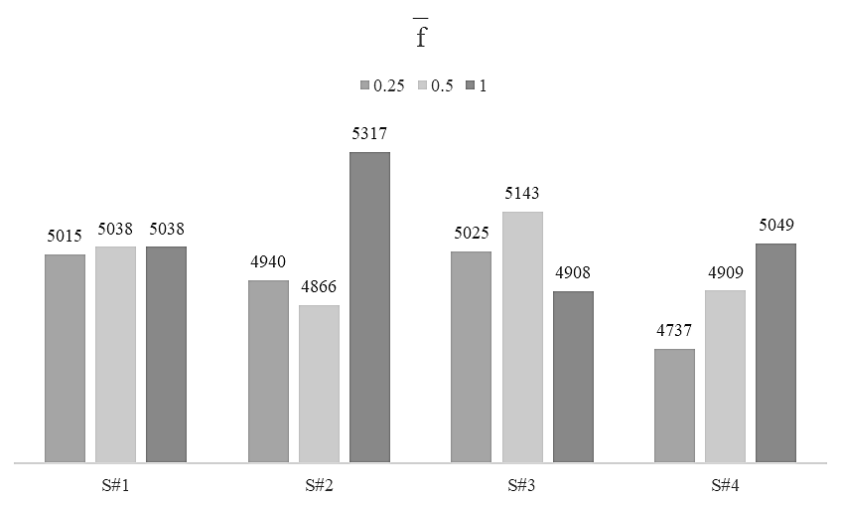

Fig 9. Variation of $\bar{f}$ with different $p_{c n r}$ values

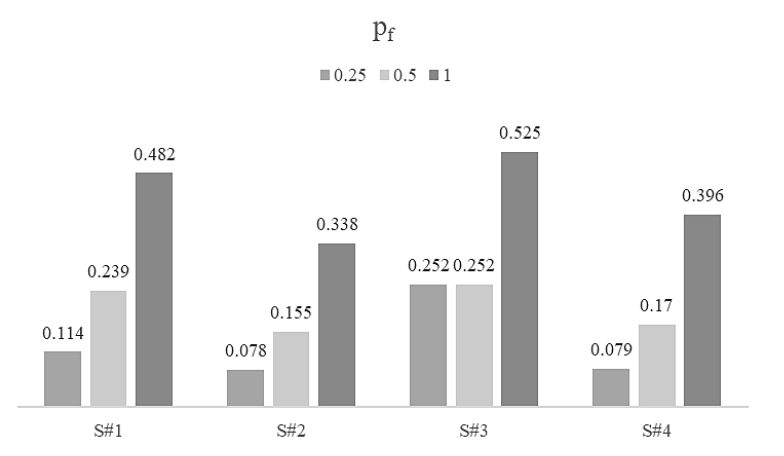

Fig 10. Variation of $p_{f}$ with different $p_{c n r}$ values

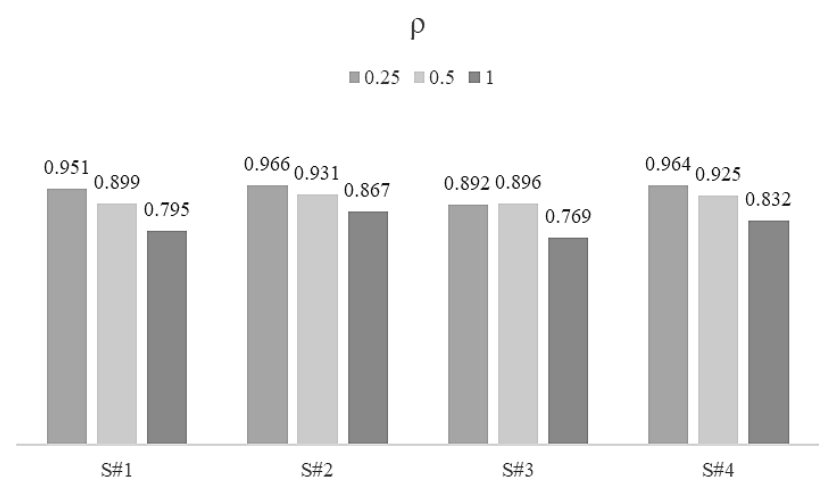

Fig 11. Variation of $\rho$ with different $p_{c n r}$ values

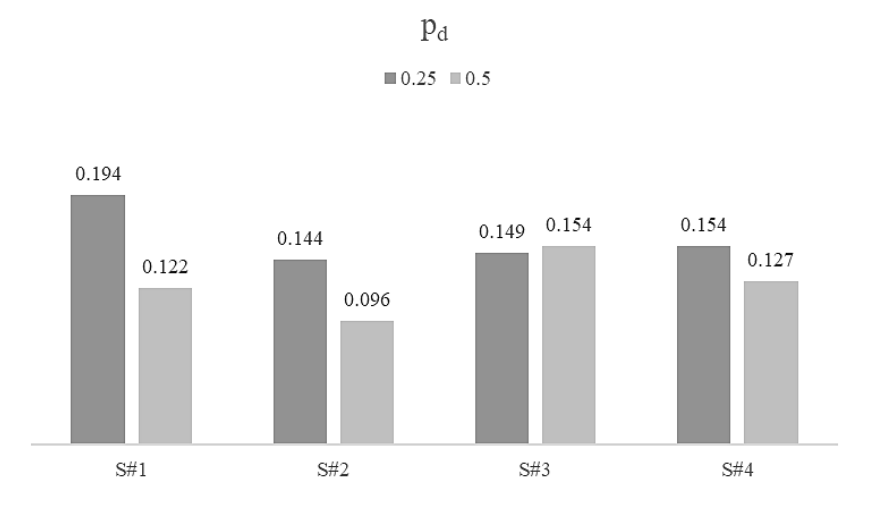

Fig 12. Variation of $p_{d}$ with different $p_{c n r}$ values

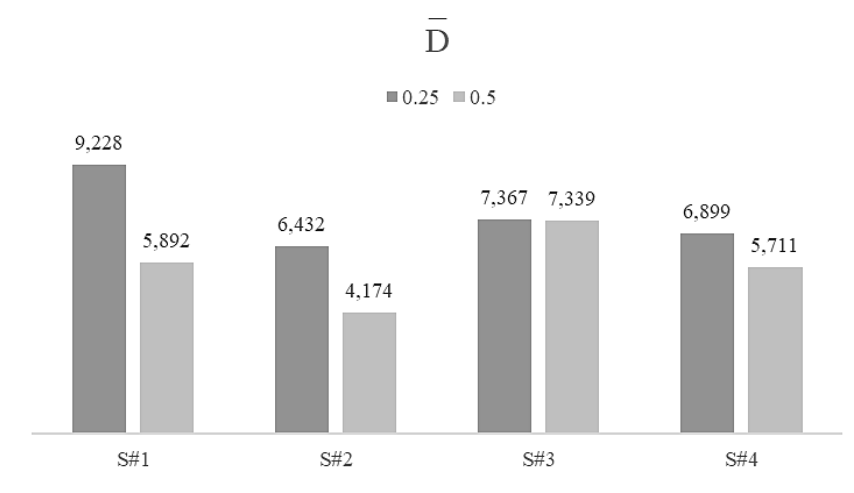

Fig 13. Variation of $\bar{D}$ with different $p_{c n r}$ values

All the configurations enhanced their probability of resilient operation, resilient operation time and resilience index with the addition of repair actions. It can be noted a decrease in probability of failure as well. It can also be observed a smaller difference between the maximum and minimum values of each parameter for all the proposed configurations. Resilient operating time, for example, exhibited variation of $46.9 \%$ and $50.5 \%$ for results in previously work and this work, respectively, as shown in Table 3, while the variation of the same parameter in Figure 8 is $5.7 \%$. It indicates that in addition to enhance the resilience of all configurations, repairing actions also improved proportionally more the systems with lower resilience, i.e., with no redundancies. It becomes clearer when metric vi is applied. The values of $p_{f}-$ $p_{d}$ are, in ascending order for $p_{c n r}=0.5: 0.242$ for $\mathrm{S} \# 2,0.270$ for S\#4, 0.359 for $S \# 1$ and 0.371 for S\#3. It can be observed that the S\#3 is the most affected system when repairing actions are taken, while $\mathrm{S \# 2}$ is the least one.

As the systems with redundancies presented greater resilience when repairing actions are disregarded, the improvement of resilience results in more simulations reaching $t_{k}=T$, i.e., until system lifetime. On the other hand, systems with no redundancies are expected to enhance their 
time until failure, although they do not reach their lifetime so often, once the lack of redundancy is a factor that has high influence in system performance. This can explain the fact that $\mathrm{S \# 2}$ and S\#4 had their time until failure hardly decreased, while S\#1 almost suffered no oscillation and S\#3 increased its value.

With the increase of repair probability, there was an increase in the number of simulations with resilient operation, since component repairing success is more likely. It is also observed the improvement of resilience index and system downtime, although the last parameter do not present significant changes in $\mathrm{S} \# 3$, which point to a system with no apparent resilience improvement. As the system with lowest resilience, $\mathrm{S} \# 3$ seemed to reach its maximum with repair actions.

Regarding resilient operating time, systems S\#1, S\#3 and S\#4 have this parameter increased. However, S\#2 decreased its value with increasing repair probability, which can indicate a more normal time operation. This conclusion is also confirmed by the time until failure, which has increased, pointing to a system with more operating time. As S\#2 is the most resilient one, it is expected that instead of enhancing its resilient operation time, the higher repair probability tends to increase its normal operation time.

Different from the results of simulations without repair, there is no convergence on which system is the most resilient according to data exhibited in Figures 7-13. With $p_{c n r}=0.5$, metris $\mathrm{i}, \mathrm{iv}, \mathrm{v}$ and vii point to the same sequence from the most to the least resilient system as found by Matelli and Goebel (2018): S\#2 -> S\#4 -> S\#1 -> S\#3. Metric ii confirms that the systems with redundancies have greater resilience values, although it was affected by the higher proportional improvement of systems with lower resilience when no repairing actions are taken.

However, iii diverges from the other metrics as it points to S\#3 and S\#1 as the most resilient ones, followed by S\#4 and $\mathrm{S \# 2}$, respectively. As discussed before, this can be caused by the increase of the number of simulations of systems with redundancies that reached lifetime.

When $p_{c n r}=0.25$, metrics $\mathrm{i}$, iv and $\mathrm{v}$ continued to point sequence S\#2 -> S\#4 -> S\#1 -> S\#3. Metric ii exhibited S\#4 as the most resilient, once $\mathrm{S} \# 2$ decreased its resilient operation time, as previously discussed. Metric iii indicated S\#3 as the one with highest resilience. The new metric vii pointed to $\mathrm{S} \# 2$ as the most resilient and $\mathrm{S} \# 1$ as the least resilient. The difference $p_{f}-p_{d}$ proposed by metric vi showed smaller values compared to when $p_{c n r}=0.5$, although S\#3 seemed to be stabilized. This fact points to a high initial impact in resilience when repairing actions are taken, followed by a reduced impact when repair probability is enhanced. This difference showed that $\mathrm{S \# 3}$ is the most affected system by repairing actions, followed by S\#1, S\#4 and $\mathrm{S} \# 2$, respectively.

\section{Conclusions}

Four cogeneration systems were developed via CLIPS and seven metrics for resilience quantification were proposed herein, with two of them considering components repairing time, a new analysis in this methodology.

With no repairment, the first five metrics pointed to the same sequence of the most to the least resilient system. Four of the seven metrics in first simulation with repair pointed to S\#2 as the most resilient system and $\mathrm{S \# 3}$ as the least resilient one. In last simulation condition, with higher repair probability, there were also four metrics considering S\#2 with higher resilience value and four of them pointing $\mathrm{S} \# 3$ with the lowest.

It became clear the importance of repairing in resilience of these systems. With repair actions, all the systems enhanced their resilience, being the higher proportional increases in those with lower resilience. In systems with no redundancy, repairment hardly improved all the values of the metrics, indicating to be a good alternative for resilience actions. This fact could be reiterated with application of metric vi, which pointed to S\#3 and S\#1 as those who suffered more impact in their resilience with the addition of repairment.

Although S\#1 exhibited higher thermal efficiency, S\#2 presented higher resilience in almost all metrics, therefore being the most resilient one. Any project that considers resilience as an important factor must focus on redundancy and repair actions, as it can be concluded by this work.

\section{ACKNOWLEDGEMENT}

To FAPESP - São Paulo Research Foundation, for financing through process 2018/02079-7.

\section{REFERENCES}

Francis, R., Bekera, B. (2014) A metric and frameworks for resilience analysis of engineered and infrastructure systems. Reliability Engineering and System Safety, vol. 121, pp. 90-103. doi: https://doi.org/10.1016/j.ress.2013.07.004

Hickford, A. J., Blainey, S. P., Hortelano, A. O., Pant, R. (2018). Resilience engineering: theory and practice in interdependent infrastructure systems. Environment Systems and Decisions, vol. 38, pp. 278-291. doi: https://doi.org/10.1007/s10669-018-9707-4

Hossain, N. U. I., Jaradat, R., Hosseini, S., Marufuzzaman, M., Buchanan, R. K. (2019). A framework for modeling and assessing system resilience using a Bayesian network: a case study of an interdependent electrical infrastructure system. International Journal of Critical Infrastructure Protection, vol. 25, pp. 62-83. doi: https://doi.org/10.1016/j.ijcip.2019.02.002

Isa, N. M., Tan, C. W., Yatim, A. H. M. (2018). A comprehensive review of cogeneration system in a microgrid: A perspective from architecture and operating system. Renewable and Sustainable Energy 
Reviews, vol. 81, pp. 2236-2263. doi: https://doi.org/10.1016/j.rser.2017.06.034

Lin, Y., Bie, Z. (2016). Study on the resilience of the integrated energy system. Energy Procedia, vol. 103, pp. 171-176. doi: 10.1016/j.egypro.2016.11.268

Matelli, J. A., Goebel, K. (2018) Conceptual design of cogeneration plants under a resilient design perspective: Resilience metrics and case study. Applied Energy, vol. 215, pp. 736-750. doi: https://doi.org/10.1016/j.apenergy.2018.02.081

Martisauskas, L., Augutis, J., Krikstolaitis, R. (2018). Methodology for energy security assessment considering energy system resilience to disruptions. Energy Strategy Reviews, vol. 22, pp. 106-118. doi: https://doi.org/10.1016/j.esr.2018.08.007

Mehrpouyan, H., Haley, B., Dong, A., Tumer, I. Y., Hoyle, C. (2015). Resiliency analysis for complex engineered system design. Artificial intelligence for engineering design, analysis and manufacturing, vol. 29, pp. 93-108. doi: 10.1017/S0890060414000663

Sandia National Laboratories (2014). Conceptual framework for developing resilience metrics for the electricity, oil, and gas sectors in the United States. Sandia Report. Springfield, United States of America: Sandia National Laboratories.

Shen, Y., Gu, C., Zhao, P. (2019). Structural vulnerability assessment of multi-energy system using a PageRank algorithm. Energy Procedia, vol. 158, pp. 6466-6471. doi: https://doi.org/10.1016/j.egypro.2019.01.132

Silva, J. C., Matelli, J. A., Bazzo, E. (2014). Development of a knowledge-based system for cogeneration plant design: Verification, validation and lessons learned. Knowledge-Based Systems, vol. 67, pp. 230-243. doi: https://doi.org/10.1016/j.knosys.2014.05.002

Yodo, N., Wang, P. (2016) Resilience allocation for early stage design of complex engineered systems. Journal of
Mechanical Design, vol. 138, pp. 1-10. doi: $10.1115 / 1.4033990$

Biographies

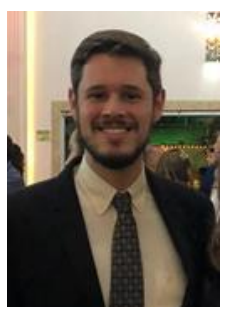

Fellipe Sartori da Silva is a second-year $\mathrm{PhD}$ student in mechanical engineering with focus on energy transmission and conversion. He obtained a bachelor degree (2015) and a master degree (2018) in mechanical engineering from São Paulo State University (UNESP) in Guaratinguetá, Brazil. He covered molten carbonate fuel cell in his master thesis and are currently studying resilience in energy systems in his doctoral dissertation. He taught transport phenomena in undergrad (2017).

José Alexandre Matelli is an associate professor at São Paulo State Universisty (UNESP), Brazil, since 2010. He obtained a bachelor degree in mechanical engineering (1998), a master degree in engineering and thermal sciences (2001, sandwich with $\mathrm{KTH}$, Sweden) and a PhD, also in engineering and thermal sciences (2008), all from Federal University of Santa Catarina, Brazil. At UNESP, he is former deputy (2013-2015) and former head (2015-2017) of the Department of Energy; currently, he is member of the Board of Trustees. He teaches heat transfer, cogeneration and alternative energy sources for mechanical engineering (undergrad) and knowledge-based systems and heat transfer for grad students. His main research interest is the application of AI in the design of thermal systems, focusing in cogeneration, fuel cells, exergy analysis, system optimization and, more recently, resilient design. In 2017, he has been a visiting scientist at NASA Ames/TI/DaSH, where he developed a framework for resilient design of complex engineering systems. 